\title{
Multi-Modal Multi sensor Interaction between Human and Heterogeneous Multi-Robot System
}

\author{
Extended Abstract \\ S M Al Mahi \\ Department of Computer Science, Oklahoma State University \\ Stillwater, Oklahoma 74078 \\ smahi@okstate.edu
}

\begin{abstract}
I introduce a novel multi-modal multi-sensor interaction method between humans and heterogeneous multi-robot systems. I have also developed a novel algorithm to control heterogeneous multirobot systems. The proposed algorithm allows the human operator to provide intentional cues and information to a multi-robot system using a multimodal multi-sensor touchscreen interface. My proposed method can effectively convey complex human intention to multiple robots as well as represent robots' intentions over the spatiotemporal domain. The proposed method is scalable and robust to dynamic change in the deployment configuration. I describe the implementation of the control algorithm used to control multiple quad-rotor unmanned aerial vehicles in simulated and real environments. I will also present my initial work on human interaction with the robots running my algorithm using mobile phone touch screens and other potential multimodal interactions.
\end{abstract}

\section{ACM Reference Format:}

S M Al Mahi. 2018. Multi-Modal Multi sensor Interaction between Human and Heterogeneous Multi-Robot System: Extended Abstract. In 2018 International Conference on Multimodal Interaction (ICMI '18), October 1620, 2018, Boulder, CO, USA. ACM, New York, NY, USA, 5 pages. https: //doi.org/10.1145/3242969.3264971

\section{INTRODUCTION}

In recent years, robots are becoming more ubiquitous and available to the general public than ever before, thanks to the rapid development of artificial intelligence and technologies like 3D printing, open-source autopilots, mission planners, and so on. Small Unmanned Aerial Systems (UAS) probably are the most common robots that people, in general, have access to today. However, there is a considerable research gap in human-UAS interaction. UAS may have been become popular for their use in photography. They have other useful applications as well. UAS have been used effectively for surveying, agriculture, search and rescue operations and so on. The means of interaction between UAS and humans has usually been joystick controllers, which can communicate control commands using radio communication mostly for the navigation intended by

Permission to make digital or hard copies of all or part of this work for personal or classroom use is granted without fee provided that copies are not made or distributed for profit or commercial advantage and that copies bear this notice and the full citation on the first page. Copyrights for components of this work owned by others than the author(s) must be honored. Abstracting with credit is permitted. To copy otherwise, or republish, to post on servers or to redistribute to lists, requires prior specific permission and/or a fee. Request permissions from permissions@acm.org.

ICMI '18, October 16-20, 2018, Boulder, CO, USA

(c) 2018 Copyright held by the owner/author(s). Publication rights licensed to ACM ACM ISBN 978-1-4503-5692-3/18/10 . \$ \$15.00

https://doi.org/10.1145/3242969.3264971 the human operator. Observation of the navigation space from the ground station or the attached camera on the UAS is critical for flight safety. However, UAS can be equipped with other sensor devices as well, to capture information such as temperature, humidity, and pressure. Although this sensory information can be crucial in many applications, it is not available via visual navigation or observation from the ground station, and cannot be performed in the most effective way using just the joystick controller.

I propose using a mobile phone touch screen as an interface between UAS (and robots in general) for these kinds of complex interactions. One of the benefits of choosing mobile phone touch screen is that many people have access to one, and thus it is the most accessible way for people, in general, to interact with robots today. However, there is an enormous research gap here as well. Most of the commercial UAS drones usually provide mobile phone applications to interact with their robots, but these applications are focused on setting up control parameters and communication channels, basic control commands, and executing predetermined maneuvers. Multimodal multi-sensory interactions are often overlooked and thus have tremendous potential for research.

The primary motivation for this work is to collect weather data using multiple UAS in a low-altitude environment. This type of task requires the use of a heterogeneous team of robots where different UAS can be equipped with different sensors. For example, flying fixed-wing UAS, which are fast but neither precise nor maneuverable, can be used to scout a large area of interest. Small quad-rotors with lower velocity but higher precision and maneuverability can be used to survey a smaller area of interest more extensively. They may also need to coordinate with ground robots for efficient deployment. Robots must coordinate with each other in general, ensuring collision avoidance among robots, a suitable spread of the UAS throughout the operating area, and deploying UAS equipped with the right sensors to investigate particular phenomena discovered in particular areas of interest, where the UAS using the specific sensor can collect essential data. I have developed a distributed algorithm to control heterogeneous teams of robots using factor graphs and loopy belief propagation. This algorithm also facilitates arbitrary human input to influence the behavior of a particular robot which is then propagated to other robots. I propose a user interface that directly visualizes the robots' intention in the space and allows the human operator to interact with it. I have already developed the fundamental functionality of the interface using a mobile web application. 


\section{RELATED WORK}

A plethora of work has been done on multi-modal communication between robots and human operators using different methods. For brevity and specificity, I highlight some of the work pertaining to UAS, drones and similar robots. Capturing and recognizing gestures of human operators has been studied in recent works $[3,12]$. Limitations and challenges of this approach have been studied in other work[4]. For example, the interaction might look rigid to an observer rather than natural, even though the movement as the gesture is natural. Another major limitation that my approach solves regarding gesture-based control is that using my method one the can provide more flexible control to the robot. Another limitation is the accuracy of detection of the gesture, especially for a novice user. Some of the above-mentioned work also used multimodal interaction with robots. Recent work [8] also presented a framework which compasses Natural User Interface (NUI) including voice, gesture, markers and a Graphical User Interface, i.e., windows, 2D graphics, 2D animations, and images. My work makes use of all these; however, I add a new perspective. In my work, robots' and human operators' belief about the world and their intentions are encoded in the GUI presentation, and I propose interaction in belief and intention space. While my proposed interface currently uses the smartphone touchscreen as the interaction medium, a NUI can also be incorporated using the accelerometer and gyroscope of the smartphone. Depth camera has been used for capturing gesture works[17] for indoor navigation.

Smart phone and tablets have been used to control drones in several works. In [10] a PC tablet was used to recognize and track down visual target for UAS. Other work[1] presented a framework for visualizing and tracking hostile drone in the airspace as an alternative to RADAR. The smartphones touch screen has been used as drone interaction interface $[6,7]$.

Prior work has used belief networks for coordinated control of multi-robot teams in the context of Markov Random Fields [2, 13, 18]. Gradient-based navigation for multi-robot navigation using potential field has been proposed by other works[9, 11]. However, these works do not incorporate human intentions and interaction. My approach not only addresses this research gap and limitations but also improves the state of the art by extending task complexity by incorporating human direction and advice.

\section{HYPOTHESIS AND TECHNICAL DESCRIPTION}

Consider a set of $n$ robots. Each of these robots $a_{i}$, where $i=1 \ldots n$, has a set of sensors $\mu_{i}$ and actuators $\alpha_{i}$. A robot has a certain policy $\pi_{i}^{t}$ at time $t$ for achieving a given task $G$ assigned for the team. When it is not part of a collaborating team, an individual robot's view of the world in the environment is a function $f$ of its sensory information and its actuators, $f\left(x_{i}\right) . f$ can be a parametric or nonparametric function. For a group of robots, it is intuitive to calculate the joint probability distribution of intention $g\left(x_{1}, \ldots, x_{n}\right)$ over the space given by Eq. 1 .

$$
g\left(x_{1}, \ldots, x_{n}\right)=\prod_{j \in J} f_{j}\left(X_{j}\right)
$$

Here, $x_{i}$ denotes the intention of robot $a_{i}, j$ are discrete index values and $X_{j}$ is a subset of intentions of all the robots and human operators who are participating in the task. The loopy belief propagation algorithm can be described over the factor representation of $g$ as

$$
\mu^{a_{v} \rightarrow a_{j}}\left(x_{v}\right)=\frac{1}{Z} \prod_{A \in N_{v} \backslash a_{j}} \mu^{A \rightarrow a_{v}}\left(x_{v}\right)
$$

Equation 2 defines the message $\mu$ passed from a variable to a factor, which consists of the normalized product of all of the messages received from the variable's neighboring factors, except for the recipient factor. Set $N_{v}$ denotes the neighboring participating robots in the team for a robot $a_{v}$.

$$
\mu^{a_{i} \rightarrow a_{v}}\left(x_{i}\right)=\sum_{x_{i}^{\prime}: x_{v}^{\prime}=x_{v}} \phi\left(x_{i}^{\prime}\right) \prod_{V \in N_{i} \backslash a_{v}} \mu^{a_{v} \rightarrow a_{i}}\left(x_{v}^{\prime}\right)
$$

Equation 3 shows the message $\mu$ passed from a factor to a variable, which is the factor function applied to the messages from all other connected variable nodes, marginalized over all of the variables except the recipient's. At a certain time $t$ a particular robot $a_{i}$ can run a optimization algorithm locally on the joint distribution of intention using its policy $\pi_{i}$.

$$
\pi_{i}^{t}=\nabla g\left(x_{1}, \ldots, x_{n}\right)
$$

Low-level controllers such as a proportionalâĂŞintegralâĂŞderivative (PID) controller can achieve the goal produced by Eq. 4. A simple gradient descent algorithm can be used for this purpose.

For human interaction, I propose that any $x_{h} \in X_{j}$ can be projected on a 2D smart phone touchscreen using a web application. The human operator can then easily interact with and tweak the parameters of the function by interacting with the phone interface.

\section{PROPOSED RESEARCH}

I propose a new interaction method between humans and a heterogeneous multi-robot team using my algorithm described in Section 3. I wish to project the joint belief space of the robots (constructed using my distributed algorithm) onto a phone's touchscreen to visualize the current beliefs of the robots and let the human interact with them in a meaningful way. This simple idea can produce an elegant solution for human interaction with a heterogeneous multi-robot team. Although the robots we are dealing with are heterogeneous (that is, they have differing sensing and actuation capabilities), we are interacting with abstract joint beliefs in intention space. This allows interaction with robots without worrying much about reprogramming the whole system to accommodate heterogeneity. The algorithm is distributed and theoretically can be scaled up to the maximum number of robots that the communication configuration can support. As mentioned earlier, research showed that touchscreen-based interaction performs better than gesture interaction. Nevertheless, all the positive aspects of gesturebased and many other multimodal sensory interaction methods can be easily integrated into the smartphone. Moreover, smartphone touchscreens are extremely accessible to today's human users.

Here I will lay out my initial work on the human interaction application. The current belief of the robot is constructed from the robot's sensory inputs and the propagated messages received from 


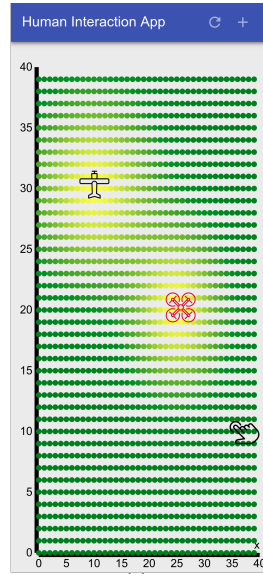

(a)

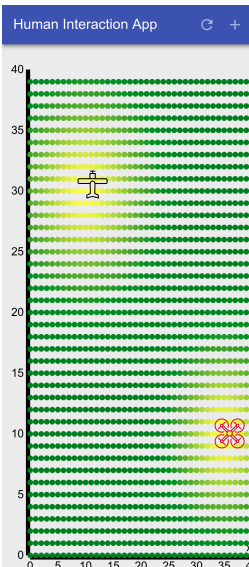

(b)

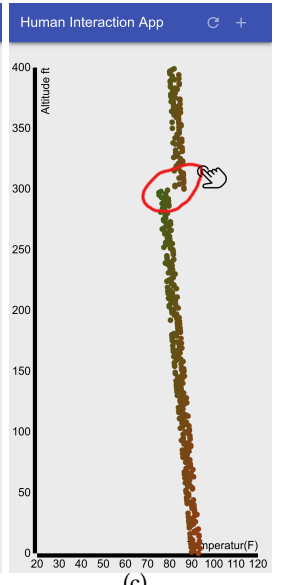

(c)
Figure 1: (a,b) A toy example where intention over the space is created as a Gaussian distribution and published as a ROS message. Human touch input directs the quadrotor away from the fixed wing aircraft. (c) A meterologist can easily determine an important temperature signature from the vertical profile provided by a quadrotor. She can mark the area of interest by sketching on intention space.

neighboring robots, and it interprets these messages based on its intention. The current belief is streamed as a ROS[14] topic and served using ROS Bridge [5] to a platform-independent Progressive Web App ${ }^{1}$. The joint intentional belief is then rendered using D3 JavaScript $^{2}$ which allows highly programmable customized visualizations and allows our approach to be more flexible in terms of designing user interactions. A snapshot of the toy demonstration of the application is shown in Fig. 1.

\section{RESEARCH PLAN}

How I am addressing important research questions poised by my hypothesis:

- Implement a distributed algorithm based on Section 1

- Implement the core functionality of the algorithm

- Experiment on the implementation using a realistic simulator, i.e. FlightGear ${ }^{3}$

- Experiment using real robots, i.e. quadrotors, fixed-wing aircraft, ground robots

- Equip different sensor packages in real robots to introduce heterogeneity

- Work with heterogeneous robots with very different flight regimes, i.e. quadrotors and fixed wing aircraft.

- Experiment with incorporating basic human input

- Multimodal multisensor human interaction

- Develop a platform-independent application for smartphones for visualizing a robot's belief

\footnotetext{
${ }^{1}$ https://developers.google.com/web/progressive-web-apps/

${ }^{2}$ https://d3js.org/

${ }^{3}$ http://home.flightgear.org/
}

- Work with robots with different sensor packages, providing live streaming of sensor data

- Implement basic human interaction using the application on touch screen

- Incorporate gyroscope and accelerometer with touch input for multimodal interaction

- Introduce sketch-like interaction with robot's belief space

- Allow interaction with multiple robots using single interaction at a time

- Experiment on simulated and real robots.

\section{RESEARCH METHODS AND PROGRESS}

So far I have implemented my belief propagation algorithm's basic functionality and experimented on simulated and real robots. Right now I am working on incorporating real fixed wing aircraft to coordinate with multiple quadrotors in the same airspace, collecting weather data and avoiding collision. I have started working on multimodal human interaction using a smart phone web application.

Experiment 1 can be described using Figure 2(a) and 2(b). Simulated UAVs A, B, and C here have an exploration task while avoiding collision and remaining within a constrained volumetric boundary - in this case, an $80 \mathrm{~m}$ cube at $5 \mathrm{~m}$ resolution, though much larger scales are algorithmically tractable. The scatter plot shows the distribution of intentions of the robots over the exploration space. In this experiment three $\phi$ factor functions are used for each robot, i.e. $\phi_{\text {unexplored }}, \phi_{\text {boundary }}$ and $\phi_{\text {avoid_collision. }} \phi_{\text {unexplored }}$ is a delta function applied to a space whenever a robot visits, making it less interesting. $\phi_{\text {boundary }}$ is a function which has a high value at the boundary of the space and zeros everywhere else. $\phi_{\text {avoid_collision }}$ is a Gaussian distribution with mean at a robot's current pose. A robot takes a normalized sum of these functions derived from both its own sensors and the messages from its neighbors to build its intention over the space. The subplot in the bottom left of Figure 2(a) shows the message received by A from C about C's intention. For a exploration task $\mathrm{C}$ has a 3D Gaussian distribution with a mean at its current sensor pose estimate. This distribution has been coded in the factor node $\phi_{c}$ of the robot. Similarly the message received from $B$ has a distribution having a mean at B's estimated current position in subplot in the middle on the left. The subplots on the right of these two subplots shows that when A sends its belief incorporating its neighbors' $(\mathrm{B}$ and $\mathrm{C}$ ) intentions using the functions defined in factor nodes $\phi_{a b}$ and $\phi_{a c}$. The subplot on the top left shows A's joint distribution of intentions incorporating its neighboring robots' messages. Figure 2(b) shows a similar process at a fixed altitude, i.e. in 2D space. It more clearly shows robot A's intention to avoid collision with robots B and C. My simulated experiment showed that UAS can explore the space without colliding with each other. I have also conducted the experiment with real robots using quadrotor UAVs (see Figure 3).

\section{CONCLUSION}

I have presented a novel model for multimodal interaction among heterogeneous multi-robot systems. I have developed the algorithm for controlling such a system and demonstrated its effectiveness using experiments with real robots and in simulation. I have presented a toy example of how this new interaction method may work 

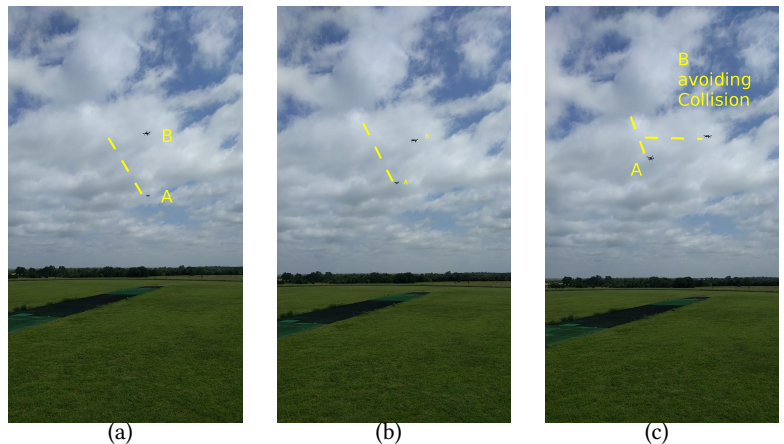

Figure 3: Experiment with two UAV robots $A$ and $B$. (a) A started moving towards B. (b) B started moving away from A's path. (c) B is avoiding collision with A.

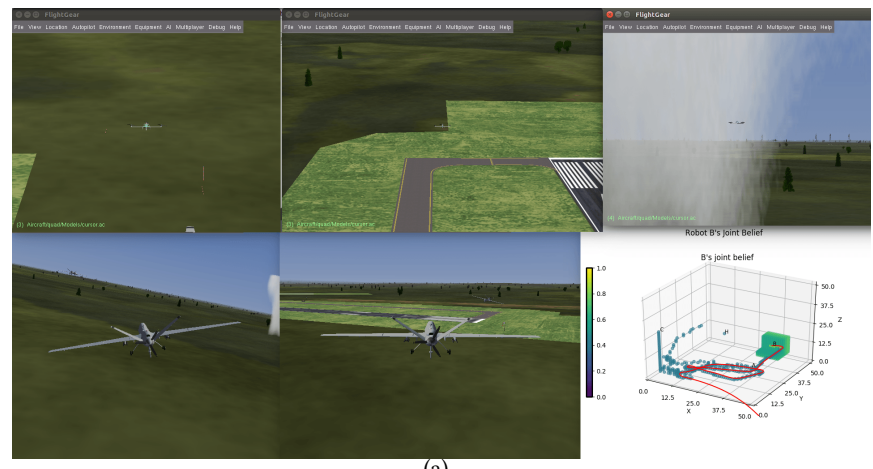

(a)

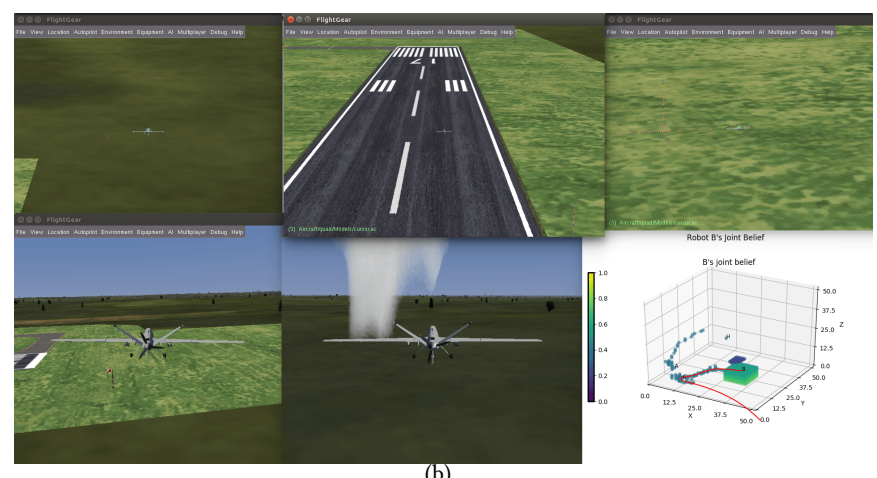

(b)

Figure 4: Snapshot of heterogeneous team exploration using Flightgear.Quadrotors and fixed-wing aircraft coordinate together to explore, and are each captured in different picture elements. The lower-right element is the joint belief of $B$. (a) UAS B is moving towards the $\mathrm{CO}_{2}$ plume. UAS A still exploring far from the plume. (b) UAS B already passed through the plume and sent intention for UAS which are interested in exploring area with high $\mathrm{CO}_{2}$ density. UAS $\mathrm{A}$ is such a robot so it moves towards the plume. B's joint belief in lower-right sub figure also shows the trail of A's path.

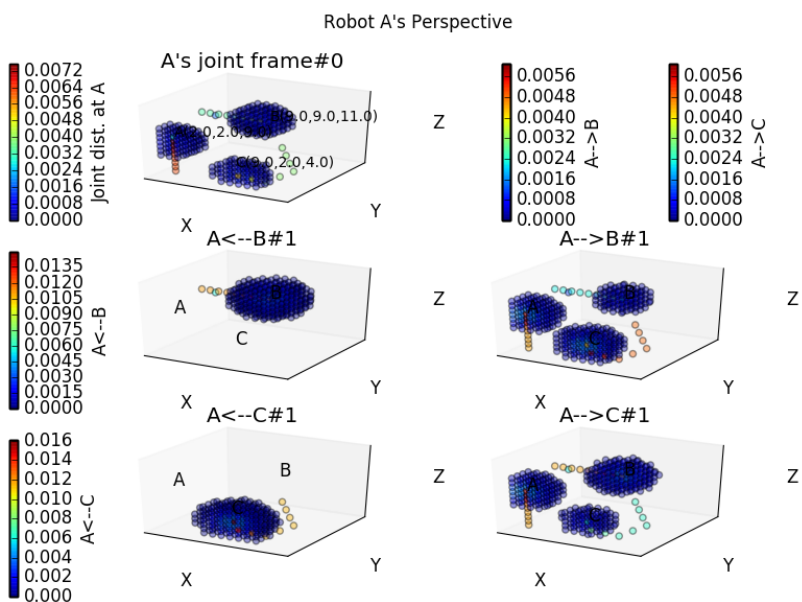

(a) Joint belief or navigation intention of simulated UAS over 3D space

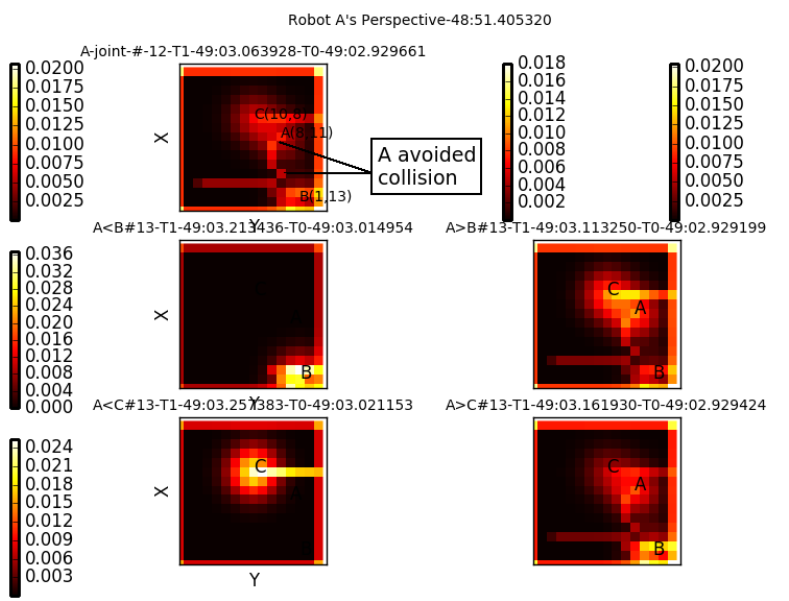

(b) Joint belief or navigation intention of simulated UAS over 2D space

Figure 2: Heat map representation of probability distribution of intention. Color bar denotes the probability mass of navigation intention over the space. $A \Rightarrow B$ denotes the intention message passed from robot $A$ to $B$. Space with higher probability mass value increases likelihood of robot choosing to visit.

and also discussed its potential. In future, I intend to implement a fully functional version of this interaction. I will add more multimodal functionality like gyroscopes, accelerometers. I plan to build a multimodal interface framework based on a smartphone touch screen that will allow users to interact seamlessly with robot teams in many possible applications.

\section{ACKNOWLEDGMENTS}

I would like to thank my advisor, Dr. Crick, for his kind support and guidance. This work is made possible through NSF grant \#1539070 (Unmanned Aircraft System for Atmospheric Physics). 


\section{REFERENCES}

[1] Sanjay K. Boddhu, Matt McCartney, Oliver Ceccopieri, and Robert L. Williams. 2013. A collaborative smartphone sensing platform for detecting and tracking hostile drones, Tien Pham, Michael A. Kolodny, and Kevin L. Priddy (Eds.), Vol. 8742. International Society for Optics and Photonics, 874211. https://doi org/10.1117/12.2014530

[2] Jesse Butterfield, Odest Chadwicke Jenkins, and Brian Gerkey. 2008. Multi-robot Markov random fields. In Autonomous Agents and Multi-Agent Systems (AAMAS).

[3] Jessica R. Cauchard, Jane L E Kevin, Y Zhai, and James A. Landay. 2015. Drone \& Me: An Exploration Into Natural Human-Drone Interaction. UbiComp '15 (2015), 361-365. https://doi.org/10.1145/2750858.2805823 arXiv:arXiv:1011.1669v3

[4] Meghan Chandarana, Erica L. Meszaros, Anna Trujillo, and B. Danette Allen. 2018. Challenges of Using Gestures in Multimodal HMI for Unmanned Mission Planning. 175-182. https://doi.org/10.1007/978-3-319-60384-1_17

[5] Christopher Crick, Graylin Jay, Sarah Osentoski, Benjamin Pitzer, and Odest Chadwicke Jenkins. 2011. Rosbridge: ROS for non-ROS users. In Proceedings of the 15th International Symposium on Robotics Research.

[6] Danielle Cummings, Stephane Fymat, and Tracy Hammond. 2012. Sketch-based Interface for Interaction with Unmanned Air Vehicles Work-in-Progress. (2012) http://delivery.acm.org/10.1145/2230000/2223664/p1511-cummings.pdf

[7] D Cummmings, S Fymat, and Tracy Hammond. 2012. RedDog : A Smart Sketch Interface for Autonomous Aerial Systems. , 21-28 pages. https://doi.org/10.2312/ SBM/SBM12/021-028

[8] Ramon A. Suarez Fernandez, Jose Luis Sanchez-Lopez, Carlos Sampedro, Hriday Bavle, Martin Molina, and Pascual Campoy. 2016. Natural user interfaces for human-drone multi-modal interaction. In 2016 Int. Conf. Unmanned Aircr. Syst. IEEE, 1013-1022. https://doi.org/10.1109/ICUAS.2016.7502665

[9] Seng Keat Gan, Robert Fitch, and Salah Sukkarieh. 2012. Real-time decentralized search with inter-agent collision avoidance. In International Conference on
Robotics and Automation (ICRA). https://doi.org/10.1109/ICRA.2012.6224975

[10] Jeongwoon Kim, David Hyunchul Shim, and James R Morrison. 2014. Tablet PC-based visual target-following system for quadrotors. 7. Intell. Robot. Syst. Theory Appl. 74, 1-2 (2014), 85-95. https://doi.org/10.1007/s10846-013-9952-1

[11] Pablo Lanillos, Seng Keat Gan, Eva Besada-Portas, Gonzalo Pajares, and Salah Sukkarieh. 2014. Multi-UAV target search using decentralized gradient-based negotiation with expected observation. Information Sciences 282 (2014), 92-110. https://doi.org/10.1016/j.ins.2014.05.054

[12] Tayyab Naseer, Jurgen Sturm, and Daniel Cremers. 2013. FollowMe: Person following and gesture recognition with a quadrocopter. In IEEE Int. Conf. Intell. Robot. Syst. IEEE, 624-630. https://doi.org/10.1109/IROS.2013.6696416

[13] Shayegan Omidshafiei, Christopher Amato, Miao Liu, Michael Everett, Jonathan P. How, and John Vian. 2017. Scalable accelerated decentralized multi-robot policy search in continuous observation spaces. In International Conference on Robotics and Automation (ICRA). https://doi.org/10.1109/ICRA.2017.7989106

[14] Morgan Quigley, Ken Conley, Brian Gerkey, Josh Faust, Tully Foote, Jeremy Leibs, Rob Wheeler, and Andrew Y Ng. 2009. ROS: an open-source Robot Operating System. In ICRA Workshop on Open Source Software.

[15] Alessandro Renzaglia, Christophe Reymann, and Simon Lacroix. 2016. Monitoring the evolution of clouds with UAVs. In International Conference on Robotics and Automation (ICRA).

[16] Christophe Reymann, Alessandro Renzaglia, Fayçal Lamraoui, Murat Bronz, and Simon Lacroix. 2017. Adaptive sampling of cumulus clouds with UAVs. Autonomous Robots (2017), 1-22.

[17] Andrea Sanna, Fabrizio Lamberti, Gianluca Paravati, and Federico Manuri. 2013. A Kinect-based natural interface for quadrotor control. Entertain. Comput. 4, 3 (aug 2013), 179-186. https://doi.org/10.1016/j.entcom.2013.01.001

[18] Ryan K Williams and Gaurav S Sukhatme. 2011. Cooperative multi-agent inference over grid structure Markov random fields. In Intelligent Robots and Systems (IROS). 